Теорія Ймовір. та Матем. Статист. Вип. 76, 2007
Theor. Probability and Math. Statist.

No. 76, 2008, Pages 169-179

S 0094-9000(08)00741-2

Article electronically published on July 17, 2008

\title{
AN ESTIMATOR FOR THE PARAMETERS OF CONCENTRATIONS OF TWO-COMPONENT MIXTURES WITH CENSORED DATA
}

\author{
UDC 519.21
}

\author{
A. YU. RIZHOV
}

\begin{abstract}
We consider a model of two-component mixtures with varying concentrations for the case of censored data. We find estimates for the concentrations of components and prove that the estimators are strongly consistent and asymptotically normal.
\end{abstract}

\section{INTRODUCTION}

The problem of estimating an unknown distribution function $F(t)$ of some random variable occurs quite often in practice when analyzing the data. Parametric and nonparametric methods are developed to estimate unknown distribution functions.

When analyzing data in biology as well as in medicine, it is often the case that there exists a certain nonnegative random variable $T$ that describes some important characteristics of the objects under consideration. The random variable $T$ is usually treated as a certain lifetime related to the objects. For example, $T$ can be the time from the onset of a disease until the death of a patient or $T$ can be the time interval between the treatment and the next relapse into disease. However, the information about the objects under observation is usually incomplete. This is the case, for example, if a patient changes his home address and this results in a gap in his file containing records for this patient. We say in these cases that the sample contains censored data and apply methods of survival analysis to estimate the distribution function of the random variable $T$ and its other characteristics (see [1, 4]).

On the other hand, the samples frequently contain objects drawn from different populations. We say that a sample contains several components in such a case. The concentration of components can be constant for all observations (the classical model of mixtures) or it can vary with observations (the model of mixtures with varying concentrations). The latter case is considered in the present paper. We show that the estimates of concentrations for two-component mixtures proposed in 5, Section 3.7] can be used in the case of samples with right-censored data. Theorem 2.1 of Section 2 claims that these estimates are strongly consistent. The asymptotic normality of these estimates is proved in Theorem 2.2. Section 3 contains some auxiliary results and proofs of the main results.

\section{Estimators of PARAMETERS OF CONCENTRATIONS AND THEIR PROPERTIES}

The problem for the model of mixtures with varying concentrations is stated as follows. Assume that $\mathfrak{X}_{1}$ and $\mathfrak{X}_{2}$ are two populations with the distribution functions of

2000 Mathematics Subject Classification. Primary 62N01; Secondary 62F12.

Key words and phrases. Mixtures with varying concentrations, censoring, Kaplan-Meier estimator. 
the lifetimes $H_{1}(t)$ and $H_{2}(t)$, respectively. A statistician considers a sample whose elements are attributed to the groups $Y_{0}, \ldots, Y_{K+1}$ according to certain characteristics. It is known that objects of the group $Y_{0}$ belong to $\mathfrak{X}_{1}$ ("positively" classified objects), while the objects of the group $Y_{K+1}$ belong to $\mathfrak{X}_{2}$ ("negatively" classified objects). The observations of other groups are not classified and may belong to either $\mathfrak{X}_{1}$ or $\mathfrak{X}_{2}$. The probability that an object of the group $j$ belongs to $\mathfrak{X}_{1}$ is denoted by $\theta_{j}$. Then the distribution of the lifetimes of objects of the group $Y_{j}$ is given by

$$
F_{j}(t)=\theta_{j} H_{1}(t)+\left(1-\theta_{j}\right) H_{2}(t), \quad t \geq 0 .
$$

By assumption, $\theta_{0} \equiv 1$ and $\theta_{K+1} \equiv 0$.

The problem is to estimate $\theta_{j}, j=1, \ldots, K$, from the sample. Mixtures whose distribution of the lifetime is given by (1) are called mixtures with varying concentrations.

Let $n_{j}$ denote the number of observations in the group $Y_{j}, n=n_{0}+\cdots+n_{K+1}$. Each object is characterized by an ordered pair of random variables $\left(T_{i j}, U_{i j}\right)$ where $T_{i j}$ is the lifetime of the object $i$ of the group $Y_{j}$ and where $U_{i j}$ is the moment of its (possible) censoring, $i=1, \ldots, n_{j}, j=0, \ldots, K+1$.

Remark 2.1. In fact, a statistician observes the variables $\min \left(T_{i j}, U_{i j}\right)$ and $\mathbb{I}_{\left\{T_{i j}<U_{i j}\right\}}$ in the case of samples with censoring, where $\mathbb{I}_{\left\{T_{i j}<U_{i j}\right\}}$ is the indicator of the event that an object $i$ of the group $j$ is censored.

Assume that the variables $T_{i j}$ and $U_{i j}$ are jointly independent and that, for a fixed $j$, have the distribution functions $F_{j}(t)=\mathrm{P}\left\{T_{i j}<t\right\}$ and $C_{j}(t)=\mathrm{P}\left\{U_{i j}<t\right\}, t \geq 0$, respectively. Note that these distribution functions do not depend on $i$. The distribution functions $F_{j}(t)$ are of the form (11) with some (unknown) concentrations $\theta_{j}$. By $\theta=\left(\theta_{1}, \ldots, \theta_{K}\right)$ we denote the vector of the true concentrations for the nonclassified groups $Y_{1}, \ldots, Y_{K}$.

Put

$$
\Pi=\left\{t \geq 0: \prod_{j=0}^{K+1}\left(1-F_{j}(t)\right)\left(1-C_{j}(t)\right)>0\right\}
$$

and fix some $\tau \in \Pi, \tau<\infty$. The distribution functions $F_{j}(t), j=0, \ldots, K+1$, and $H_{i}(t), i=1,2$, are assumed to be constant outside the interval $[0, \tau]$. Similarly to [5, Section 3.7] one can prove that $\theta$ is a point of maximum in $\alpha$ of the function

$$
R_{n}(\alpha)=\frac{\alpha_{*}^{T} B_{n} \alpha_{*}}{\alpha_{*}^{T} S_{n} \alpha_{*}}=\frac{\left(\theta_{*}^{T} S_{n} \alpha_{*}\right)^{2}}{\alpha_{*}^{T} S_{n} \alpha_{*}} \int_{0}^{\tau}\left(H_{1}(t)-H_{2}(t)\right)^{2} \lambda(d t)
$$

considered in the set $\Theta=\left\{\alpha=\left(\alpha_{1}, \ldots, \alpha_{K}\right)^{T}: 0 \leq \alpha_{i} \leq 1, i=1, \ldots, K\right\}$. Here $B_{n}=\left(b_{l m}^{n}\right)$ and $S_{n}=\left(s_{l m}^{n}\right)$ are $(K+1) \times(K+1)$ matrices with the entries

$$
b_{l m}^{n}=\sum_{i, j=0}^{K+1} \frac{n_{i} n_{j}}{n^{2}} \int_{0}^{\tau} F_{i}(t) F_{j}(t)\left(\mathbb{I}_{\{l=i\}}-\frac{n_{l}}{n}\right)\left(\mathbb{I}_{\{m=j\}}-\frac{n_{m}}{n}\right) \lambda(d t)
$$

and

$$
s_{l m}^{n}=\frac{n_{l}}{n}\left(\mathbb{I}_{\{l=m\}}-\frac{n_{m}}{n}\right),
$$

respectively, $\lambda$ is the Lebesgue measure on $[0, \tau], \alpha_{*}=\left(\alpha_{0}, \alpha_{1}, \ldots, \alpha_{K}\right)^{T}$ is an extended vector $\alpha$ with the first component $\alpha_{0}=1$, and $\mathbb{I}_{A}$ is the indicator of the set $A$. The function $R_{n}(\alpha)$ is called the theoretical contrast.

Since $R_{n}(\alpha)$ depends on the unknown functions $F_{j}(t), j=0, \ldots, K+1$, it cannot be used to construct an estimator for $\theta$. Instead, one can use the estimates $\hat{F}_{j}(t)=1-\hat{S}_{j}(t)$ 
of the functions themselves, where $\hat{S}_{j}(t)$ is the Kaplan-Meier estimator constructed from $Y_{j}$ [1, 2]. As a result, we obtain the so-called empirical contrast

$$
\hat{R}_{n}(\alpha)=\frac{\alpha_{*}^{T} \hat{B}_{n} \alpha_{*}}{\alpha_{*}^{T} S_{n} \alpha_{*}}
$$

where $\hat{B}_{n}=\left(\hat{b}_{l m}^{n}\right)$ is the $(K+1) \times(K+1)$ matrix with the random entries

$$
\hat{b}_{l m}^{n}=\sum_{i, j=0}^{K+1} \frac{n_{i} n_{j}}{n^{2}} \int_{0}^{\tau} \hat{F}_{i}(t) \hat{F}_{j}(t)\left(\mathbb{I}_{\{l=i\}}-\frac{n_{l}}{n}\right)\left(\mathbb{I}_{\{m=j\}}-\frac{n_{m}}{n}\right) \lambda(d t)
$$

and where the estimator $\hat{\theta}_{n}$ of the parameter $\theta$ is defined as the maximum of the empirical contrast with respect to $\alpha \in \Theta$.

Theorem 2.1 (Strong consistency of $\hat{\theta}_{n}$ ). Assume that

(1) the limits

$$
\lim _{n \rightarrow \infty} \frac{n_{j}}{n}=h_{j}>0
$$

exist for all $j=0, \ldots, K+1$;

(2) there exists $t \in[0, \tau]$ such that $H_{1}(t) \neq H_{2}(t)$.

Then the estimator $\hat{\theta}_{n}$ is strongly consistent, that is, $\rho\left(\hat{\theta}_{n}, \theta\right) \rightarrow 0$ almost surely as $n \rightarrow \infty$ where $\rho$ is the Euclidean metric in $\Theta$.

A useful procedure to evaluate the estimator of the vector $\theta$ can be described as follows. First we find the maximal eigenvalue $\lambda^{\max }$ of the matrix $S_{n}^{-1} \hat{B}_{n}$ (note that the matrix $S_{n}$ is nondegenerate for sufficiently large $n$ ). Then we choose an arbitrary eigenvector $\alpha_{*}^{\prime}=\left(\alpha_{0}^{\prime}, \ldots, \alpha_{K}^{\prime}\right)^{T}$ corresponding to the eigenvalue $\lambda^{\max }$. If $\alpha_{0}^{\prime} \neq 0$, then the projection of the vector

$$
\left(\alpha_{1}^{\prime} / \alpha_{0}^{\prime}, \ldots, \alpha_{K}^{\prime} / \alpha_{0}^{\prime}\right)^{T}
$$

to the set $\Theta$ can be considered as an estimator for $\theta$. According to Theorem 2.1, the probability that this estimator coincides with the point of maximum of the empirical contrast $\hat{R}_{n}(\alpha)$ tends to zero.

Let

$$
R(\theta)=\left(\theta_{*}^{T} S \theta_{*}\right) \int_{0}^{\tau}\left(H_{1}(t)-H_{2}(t)\right)^{2} \lambda(d t),
$$

where the matrix $S=\left(s_{l m}\right)$ is the limit of the sequence of matrices $\left\{S_{n}, n \geq 1\right\}$. Let $Q(\theta)=\left(q_{k l}(\theta)\right)_{k, l=1}^{K}$ be the matrix with the entries

$$
q_{k l}(\theta)=\frac{2 R(\theta)}{\left(\theta_{*}^{T} S \theta_{*}\right)^{2}}\left(\sum_{i, j=0}^{K} \theta_{i}\left(s_{l i} s_{k j}-s_{i j} s_{l k}\right) \theta_{j}\right) .
$$

Theorem 2.2 (The asymptotic normality of $\hat{\theta}_{n}$ ). Let the functions $F_{j}(t)$ and $C_{j}(t)$, $t \geq 0$, be continuous in the interval $[0, \tau]$ for all $j=0, \ldots, K+1$. If the assumptions of Theorem 2.1 hold, then

$$
\sqrt{n}\left(\hat{\theta}_{n}-\theta\right) \Longrightarrow Q^{-1}(\theta) \zeta(\theta), \quad n \rightarrow \infty,
$$

where the vector $\zeta(\theta)=\left(\zeta_{1}(\theta), \ldots, \zeta_{K}(\theta)\right)^{T}$ is Gaussian with zero mean vector (here and in what follows the symbol $\Longrightarrow$ stands for weak convergence). 


\section{Proofs of the Results}

The strong consistency of the estimators. The proof of Theorem 2.1 is based on the following auxiliary result.

Lemma 3.1. If the assumptions of Theorem 2.1 hold, then

$$
\sup _{\alpha \in \Theta}\left|\hat{R}_{n}(\alpha)-R_{n}(\alpha)\right| \rightarrow 0 \quad \text { almost surely }
$$

as $n \rightarrow \infty$.

Proof. For a fixed $\alpha \in \Theta$, we obtain from the first equality in (2) that

$$
\hat{R}_{n}(\alpha)-R_{n}(\alpha)=\frac{\alpha_{*}^{T} \hat{B}_{n} \alpha_{*}}{\alpha_{*}^{T} S_{n} \alpha_{*}}-\frac{\alpha_{*}^{T} B_{n} \alpha_{*}}{\alpha_{*}^{T} S_{n} \alpha_{*}}=\frac{1}{\alpha_{*}^{T} S_{n} \alpha_{*}}\left(\alpha_{*}^{T}\left(\hat{B}_{n}-B_{n}\right) \alpha_{*}\right) .
$$

The entries of the matrix $S_{n}$ converge to those of the limit matrix

$$
S=\left(s_{l m}\right)
$$

as $n \rightarrow \infty$. Moreover

$$
s_{l m}=\lim _{n \rightarrow \infty} s_{l m}^{n}=\lim _{n \rightarrow \infty} \frac{n_{l}}{n}\left(\mathbb{I}_{\{l=m\}}-\frac{n_{m}}{n}\right)=h_{l} \mathbb{I}_{\{l=m\}}-h_{l} h_{m} .
$$

This implies that

$$
\alpha_{*}^{T} S_{n} \alpha_{*} \rightarrow \alpha_{*}^{T} S \alpha_{*}, \quad n \rightarrow \infty
$$

uniformly in $\alpha$.

The matrix $S$ is symmetric. By the Sylvester criterion, it is positive definite; thus the quadratic form $\alpha_{*}^{T} S \alpha_{*}$ is nonnegative and is equal to zero if and only if $\alpha_{*}=0$. The latter equality is not possible, since the first component of the extended vector $\alpha_{*}$ equals 1 by definition.

Thus $\alpha_{*}^{T} S \alpha_{*}>0$ for all $\alpha \in \Theta$. An analogous result with $n_{i} / n$ instead of $h_{i}$ holds for the quadratic form $\alpha_{*}^{T} S_{n} \alpha_{*}$ for all $n$ starting with some number $n_{0}^{\prime}$. Therefore there exists a constant $C$ such that

$$
\inf _{\alpha \in \Theta} \alpha_{*}^{T} S_{n} \alpha_{*} \geq C>0, \quad n \geq n_{0}^{\prime} .
$$

It remains to show that $\sup _{\alpha \in \Theta}\left|\alpha_{*}^{T}\left(\hat{B}_{n}-B_{n}\right) \alpha_{*}\right| \rightarrow 0$ almost surely as $n \rightarrow \infty$. By the definition of the entries of the matrices $\hat{B}_{n}$ and $B_{n}$,

$$
\hat{b}_{l m}^{n}-b_{l m}^{n}=\sum_{i, j=0}^{K+1} \frac{n_{i} n_{j}}{n^{2}} \int_{0}^{\tau}\left(\hat{F}_{i}(t) \hat{F}_{j}(t)-F_{i}(t) F_{j}(t)\right)\left(\mathbb{I}_{\{l=i\}}-\frac{n_{l}}{n}\right)\left(\mathbb{I}_{\{m=j\}}-\frac{n_{m}}{n}\right) \lambda(d t)
$$

for all $l, m=0, \ldots, K+1$, whence

$$
\begin{aligned}
\left|\hat{b}_{l m}^{n}-b_{l m}^{n}\right| & \leq\left(\sum_{i, j=0}^{K+1} \int_{0}^{\tau}\left|\hat{F}_{i}(t) \hat{F}_{j}(t)-F_{i}(t) F_{j}(t)\right| \lambda(d t)\right) \\
& \leq \sum_{i, j=0}^{K+1}\left(\int_{0}^{\tau}\left|\hat{F}_{j}(t)-F_{j}(t)\right| \lambda(d t)+\int_{0}^{\tau}\left|\hat{F}_{i}(t)-F_{i}(t)\right| \lambda(d t)\right) \\
& \leq \lambda([0, \tau]) \sum_{i, j=0}^{K+1}\left(\sup _{t \in[0, \tau]}\left|\hat{F}_{j}(t)-F_{j}(t)\right|+\sup _{t \in[0, \tau]}\left|\hat{F}_{i}(t)-F_{i}(t)\right|\right) \rightarrow 0
\end{aligned}
$$

almost surely, since the corresponding distribution functions and their estimators are bounded for all $i$ and $j$. The convergence above holds in view of [6, Theorem 1] where the strong consistency of the Kaplan-Meier estimator is proved. Since $\Theta$ is a compact 
set, we have $\sup _{\alpha \in \Theta}\left|\alpha_{*}^{T}\left(\hat{B}_{n}-B_{n}\right) \alpha_{*}\right| \rightarrow 0$ almost surely as $n \rightarrow \infty$. This observation completes the proof of the lemma.

Proof of Theorem 2.1. The second equality in (2) implies that

$$
R_{n}(\alpha)=\frac{\left(\theta_{*}^{T} S_{n} \alpha_{*}\right)^{2}}{\alpha_{*}^{T} S_{n} \alpha_{*}} \int_{0}^{\tau}\left(H_{1}(t)-H_{2}(t)\right)^{2} \lambda(d t) .
$$

Hence

$$
0 \leq R_{n}(\theta)-R_{n}\left(\hat{\theta}_{n}\right)=\left(\left(\theta_{*}^{T} S_{n} \theta_{*}\right)-\frac{\left(\theta_{*}^{T} S_{n}\left(\hat{\theta}_{n}\right)_{*}\right)^{2}}{\left(\hat{\theta}_{n}\right)_{*}^{T} S_{n}\left(\hat{\theta}_{n}\right)_{*}}\right) \int_{0}^{\tau}\left(H_{1}(t)-H_{2}(t)\right)^{2} \lambda(d t) .
$$

On the other hand, Lemma 3.1 implies that for all $\varepsilon>0$ there exists an integer number $n_{0}^{\prime \prime}$ such that

$$
\sup _{\alpha \in \Theta}\left|\hat{R}_{n}(\alpha)-R_{n}(\alpha)\right|<\varepsilon, \quad n \geq n_{0}^{\prime \prime},
$$

almost surely, whence

$0 \leq R_{n}(\theta)-R_{n}\left(\hat{\theta}_{n}\right)=\left(R_{n}(\theta)-\hat{R}_{n}(\theta)\right)+\left(\hat{R}_{n}(\theta)-\hat{R}_{n}\left(\hat{\theta}_{n}\right)\right)+\left(\hat{R}_{n}\left(\hat{\theta}_{n}\right)-R_{n}\left(\hat{\theta}_{n}\right)\right) \leq 2 \varepsilon$,

since $\hat{\theta}_{n}$ is a point of maximum of the empirical contrast $\hat{R}_{n}(\alpha)$. Thus

$$
0 \leq\left(\theta_{*}^{T} S_{n} \theta_{*}\right)\left(1-\frac{\left(\theta_{*}^{T} S_{n}\left(\hat{\theta}_{n}\right)_{*}\right)^{2}}{\left(\theta_{*}^{T} S_{n} \theta_{*}\right)\left(\left(\hat{\theta}_{n}\right)_{*}^{T} S_{n}\left(\hat{\theta}_{n}\right)_{*}\right)}\right) \int_{0}^{\tau}\left(H_{1}(t)-H_{2}(t)\right)^{2} \lambda(d t) \leq 2 \varepsilon
$$

for $n \geq n_{0}^{\prime \prime}$.

By the assumptions of the theorem, the integral is not equal to zero. Relation (6) implies that $\theta_{*}^{T} S_{n} \theta_{*} \geq C>0$ for all $n \geq \max \left(n_{0}^{\prime}, n_{0}^{\prime \prime}\right)$; thus

$$
1-\frac{\left(\theta_{*}^{T} S_{n}\left(\hat{\theta}_{n}\right)_{*}\right)^{2}}{\left(\theta_{*}^{T} S_{n} \theta_{*}\right)\left(\hat{\theta}_{n}\right)_{*}^{T} S_{n}\left(\hat{\theta}_{n}\right)_{*}} \rightarrow 0, \quad n \rightarrow \infty,
$$

almost surely.

The set $\Theta$ is compact and the function

$$
f(\alpha)=1-\frac{\left(\theta_{*}^{T} S \alpha_{*}\right)^{2}}{\left(\theta_{*}^{T} S \theta_{*}\right)\left(\alpha_{*}^{T} S \alpha_{*}\right)}
$$

is continuous in $\Theta$. Moreover $f(\alpha)=0$ if and only if $\alpha=\theta$, since the first components of the vectors $\theta_{*}$ and $\alpha_{*}$ are equal to 1 by definition. This completes the proof of the theorem.

The asymptotic normality of the estimators. Both the theoretical and empirical contrasts are continuous with respect to $\alpha$ and $\alpha_{*}^{T} S_{n} \alpha_{*} \geq C>0$ for all $n \geq 1$. Thus the derivatives

exist for all $k, l=1, \ldots, K$.

$$
\frac{\partial R_{n}(\alpha)}{\partial \alpha_{k}}, \quad \frac{\partial \hat{R}_{n}(\alpha)}{\partial \alpha_{k}}, \quad \frac{\partial^{2} R_{n}(\alpha)}{\partial \alpha_{k} \partial \alpha_{l}}, \quad \frac{\partial^{2} \hat{R}_{n}(\alpha)}{\partial \alpha_{k} \partial \alpha_{l}}
$$

Lemma 3.2. Let $\theta \in \Theta$ be the true value of the vector of concentrations and let $\hat{\theta}_{n}$ be its estimator. Assume that the vector $\xi_{n}=\hat{\theta}_{n}+\tau_{n}\left(\theta-\hat{\theta}_{n}\right)$ is such that

$$
\hat{R}_{n}^{\prime}\left(\hat{\theta}_{n}\right)-\hat{R}_{n}^{\prime}(\theta)=\hat{R}_{n}^{\prime \prime}\left(\xi_{n}\right)\left(\hat{\theta}_{n}-\theta\right)
$$


for some $\tau_{n} \in(0,1)$. Then there exists a sequence of random variables $\left\{\gamma_{n}, n \geq 1\right\}$ such that $\gamma_{n} \rightarrow 0$ almost surely as $n \rightarrow \infty$ and

$$
\left\|\hat{R}_{n}^{\prime \prime}\left(\xi_{n}\right)-Q(\theta)\right\| \leq \gamma_{n}, \quad n \geq n_{0}^{\prime},
$$

almost surely where $Q(\theta)=\lim _{n \rightarrow \infty} R_{n}^{\prime \prime}(\theta)$ is a nondegenerate matrix.

Remark 3.1. The convergence of matrices means that all their entries converge.

Proof. First we show that

$$
\sup _{\alpha \in \Theta}\left|\frac{\partial^{2}}{\partial \alpha_{k} \partial \alpha_{l}} \hat{R}_{n}(\alpha)-\frac{\partial^{2}}{\partial \alpha_{k} \partial \alpha_{l}} R_{n}(\alpha)\right| \rightarrow 0, \quad n \rightarrow \infty,
$$

almost surely for all $k, l=1, \ldots, K$.

Put

$$
\Lambda_{n}(\alpha)=\alpha_{*}^{T} \hat{B}_{n} \alpha-\alpha_{*}^{T} B_{n} \alpha=\alpha_{*}^{T}\left(\hat{B}_{n}-B_{n}\right) \alpha_{*} .
$$

It follows from (5) and (마) that

$$
\left|\frac{\partial}{\partial \alpha_{k}}\left(\frac{1}{\Delta_{n}(\alpha)}\right)\right|<\infty, \quad\left|\frac{\partial^{2}}{\partial \alpha_{k} \partial \alpha_{l}}\left(\frac{1}{\Delta_{n}(\alpha)}\right)\right|<\infty
$$

for all $\alpha$ and $n \geq n_{0}^{\prime}$, where $n_{0}^{\prime}$ is a certain integer number and where $\Delta_{n}(\alpha)=\alpha_{*}^{T} S_{n} \alpha_{*}$. Then

$$
\begin{aligned}
\frac{\partial^{2} \hat{R}_{n}(\alpha)}{\partial \alpha_{k} \partial \alpha_{l}}-\frac{\partial^{2} R_{n}(\alpha)}{\partial \alpha_{k} \partial \alpha_{l}}= & \frac{\partial^{2} \Lambda_{n}(\alpha)}{\partial \alpha_{k} \partial \alpha_{l}} \frac{1}{\Delta_{n}(\alpha)}+\frac{\partial \Lambda_{n}(\alpha)}{\partial \alpha_{k}} \frac{\partial}{\partial \alpha_{l}}\left(\frac{1}{\Delta_{n}(\alpha)}\right) \\
& +\Lambda_{n}(\alpha) \frac{\partial^{2}}{\partial \alpha_{k} \partial \alpha_{l}}\left(\frac{1}{\Delta_{n}(\alpha)}\right)+\frac{\partial \Lambda_{n}(\alpha)}{\partial \alpha_{l}} \frac{\partial}{\partial \alpha_{k}}\left(\frac{1}{\Delta_{n}(\alpha)}\right) .
\end{aligned}
$$

Since

$$
\frac{\partial \Lambda_{n}(\alpha)}{\partial \alpha_{k}}=2 \sum_{j=0}^{K}\left(\hat{b}_{j k}^{n}-b_{j k}^{n}\right) \alpha_{j}, \quad \frac{\partial^{2} \Lambda_{n}(\alpha)}{\partial \alpha_{k} \partial \alpha_{l}}=2\left(\hat{b}_{k l}^{n}-b_{k l}^{n}\right)
$$

and

$$
\left|\hat{b}_{k l}^{n}-b_{k l}^{n}\right| \rightarrow 0, \quad n \rightarrow \infty,
$$

almost surely for all $k$ and $l$, relation (17) holds.

Now we evaluate the matrix $R_{n}^{\prime \prime}(\alpha)$ of the second derivatives at the point $\alpha=\theta$. We have for $k, l=1, \ldots, K$ that

$$
\left.\frac{\partial^{2} R_{n}(\alpha)}{\partial \alpha_{k} \partial \alpha_{l}}\right|_{\alpha=\theta}=\left.\int_{0}^{\tau}\left(H_{1}(t)-H_{2}(t)\right)^{2} \lambda(d t) \frac{\partial^{2}}{\partial \alpha_{k} \partial \alpha_{l}}\left(\frac{\left(\theta_{*}^{T} S_{n} \alpha_{*}\right)^{2}}{\alpha_{*}^{T} S_{n} \alpha_{*}}\right)\right|_{\alpha=\theta} .
$$

Since $\partial R_{n}(\alpha) /\left.\partial \alpha_{k}\right|_{\alpha=\theta}=0$ for all $k$, the simple algebra yields

$$
\left.\frac{\partial^{2} R_{n}(\alpha)}{\partial \alpha_{k} \partial \alpha_{l}}\right|_{\alpha=\theta}=\frac{2}{\Delta_{n}(\theta)} \int_{0}^{\tau}\left(H_{1}(t)-H_{2}(t)\right)^{2} \lambda(d t)\left(\sum_{i, j=0}^{K} \theta_{i}\left(s_{l i}^{n} s_{k j}^{n}-s_{i j}^{n} s_{l k}^{n}\right) \theta_{j}\right) .
$$

The limit contrast

$$
R(\alpha)=\frac{\left(\theta_{*}^{T} S \alpha_{*}\right)^{2}}{\alpha_{*}^{T} S \alpha_{*}} \int_{0}^{\tau}\left(H_{1}(t)-H_{2}(t)\right)^{2} \lambda(d t)
$$

exists for all $\alpha \in \Theta$ as $n \rightarrow \infty$ by (2) and (5). By the same reason, the entry $(k, l)$ of the matrix $Q(\theta)$ is of the following form:

$$
q_{k l}(\theta)=\frac{2 R(\theta)}{\Delta^{2}(\theta)}\left(\sum_{i, j=0}^{K} \theta_{i}\left(s_{l i} s_{k j}-s_{i j} s_{l k}\right) \theta_{j}\right) .
$$


Thus

$$
\begin{aligned}
& \left|\frac{\partial^{2} \hat{R}_{n}(\alpha)}{\partial \alpha_{k} \partial \alpha_{l}}\right|_{\alpha=\xi_{n}}-q_{k l}(\theta) \mid \\
& \leq\left|\frac{\partial^{2} \hat{R}_{n}(\alpha)}{\partial \alpha_{k} \partial \alpha_{l}}\right|_{\alpha=\xi_{n}}-\left.\left.\frac{\partial^{2} R_{n}(\alpha)}{\partial \alpha_{k} \partial \alpha_{l}}\right|_{\alpha=\xi_{n}}|+| \frac{\partial^{2} R_{n}(\alpha)}{\partial \alpha_{k} \partial \alpha_{l}}\right|_{\alpha=\xi_{n}}-\left.\frac{\partial^{2} R_{n}(\alpha)}{\partial \alpha_{k} \partial \alpha_{l}}\right|_{\alpha=\theta} \mid \\
& \quad+\left|\frac{\partial^{2} R_{n}(\alpha)}{\partial \alpha_{k} \partial \alpha_{l}}\right|_{\alpha=\theta}-q_{k l}(\theta) \mid \\
& \leq \sup _{\alpha \in \Theta}\left|\frac{\partial^{2} \hat{R}_{n}(\alpha)}{\partial \alpha_{k} \partial \alpha_{l}}-\frac{\partial^{2} R_{n}(\alpha)}{\partial \alpha_{k} \partial \alpha_{l}}\right|+\sup _{t \in[0,1]}\left|\frac{\partial^{2} R_{n}(\alpha)}{\partial \alpha_{k} \partial \alpha_{l}}\right|_{\alpha=\hat{\theta}_{n}+t\left(\theta-\hat{\theta}_{n}\right)}-\left.\frac{\partial^{2} R_{n}(\alpha)}{\partial \alpha_{k} \partial \alpha_{l}}\right|_{\alpha=\theta} \mid \\
& \quad+\left|\frac{\partial^{2} R_{n}(\alpha)}{\partial \alpha_{k} \partial \alpha_{l}}\right|_{\alpha=\theta}-q_{k l}(\theta) \mid
\end{aligned}
$$

almost surely for all $k$ and $l$.

Denote the latter sum by $\gamma_{n}^{k l}$. Each of the terms approaches zero, whence $\gamma_{n}^{k l} \rightarrow 0$ as $n \rightarrow \infty$. Putting

$$
\gamma_{n}=\sqrt{\sum_{l, k=1}^{K}\left(\gamma_{n}^{k l}\right)^{2}}
$$

we complete the proof of the lemma.

Remark 3.2. The matrix $Q(\theta)$ is nondegenerate by [5, Lemma 3.5.2].

Proof of Theorem 2.2. Lemma 3.2 implies that

$$
\sqrt{n}\left(\hat{\theta}_{n}-\theta\right)=-\left(\hat{R}_{n}^{\prime \prime}\left(\xi_{n}\right)\right)^{-1} \sqrt{n}\left(\hat{R}_{n}^{\prime}(\theta)-\hat{R}_{n}^{\prime}\left(\hat{\theta}_{n}\right)\right) .
$$

Moreover $\left\|\hat{R}_{n}^{\prime \prime}\left(\xi_{n}\right)-Q(\theta)\right\| \leq \gamma_{n} \rightarrow 0$ almost surely as $n \rightarrow \infty$ and the matrix $Q(\theta)$ is nonrandom and nondegenerate. On the other hand, $\hat{R}_{n}^{\prime}\left(\hat{\theta}_{n}\right)=R_{n}^{\prime}(\theta)=0$; hence

$$
\sqrt{n}\left(\hat{R}_{n}^{\prime}(\theta)-\hat{R}_{n}^{\prime}\left(\hat{\theta}_{n}\right)\right)=\sqrt{n}\left(\hat{R}_{n}^{\prime}(\theta)-R_{n}^{\prime}(\theta)\right) .
$$

It remains to obtain the asymptotic behavior of the right-hand side of the latter equality at the point $\theta$. We use another representation of the theoretical and empirical contrasts. For an arbitrary vector $a=\left(a_{0}, \ldots, a_{K+1}\right)^{T}$ let

$$
\langle a\rangle_{n}=\sum_{j=0}^{K+1} \frac{n_{j}}{n} a_{j}
$$

The product of two vectors $a$ and $b$ of the same dimension $(K+2) \times 1$ is understood as the vector

$$
a b=\left(a_{0} b_{0}, \ldots, a_{K+1} b_{K+1}\right)^{T} .
$$

Let the vector $u_{n}(\alpha)$ be defined by its components:

$$
u_{j}^{n}(\alpha)=\frac{1}{\sqrt{\Delta_{n}(\alpha)}} \sum_{l=0}^{K} \alpha_{l}\left(\mathbb{I}_{\{l=j\}}-\frac{n_{l}}{n}\right), \quad j=0, \ldots, K+1 .
$$

Note that

$$
\begin{aligned}
\left\langle u_{n}(\alpha)\right\rangle_{n} & =0, \\
\left\langle u_{n}^{2}(\alpha)\right\rangle_{n} & =1 .
\end{aligned}
$$


Put $F(t)=\left(F_{0}(t), \ldots, F_{K+1}(t)\right)^{T}, t \in[0, \tau]$. Then

$$
R_{n}(\alpha)=\int_{0}^{\tau}\left(\left\langle F(t) u_{n}(\alpha)\right\rangle_{n}\right)^{2} \lambda(d t)
$$

(see [5, Section 3.1]). A similar expression holds for the empirical contrast, too (if one substitutes the estimator $\hat{F}_{j}(t)$ for the distribution function $F_{j}(t)$ in the above formula).

The derivatives $\left(\partial / \partial \alpha_{k}\right) u_{j}^{n}(\alpha)$ exist and are bounded for all $j$ and $k$ by (6) . Thus

$$
\frac{\partial R_{n}(\alpha)}{\partial \alpha_{k}}=2 \int_{0}^{\tau}\left\langle F(t) u_{n}(\alpha)\right\rangle_{n}\left\langle\frac{\partial u_{n}(\alpha)}{\partial \alpha_{k}} F(t)\right\rangle_{n} \lambda(d t)
$$

where $\partial u_{n}(\alpha) / \partial \alpha_{k}$ is the vector of the first partial derivatives with respect to $\alpha_{k}$. It is easy to check that

$$
\begin{aligned}
\sqrt{n}( & \left.\frac{\partial \hat{R}_{n}(\alpha)}{\partial \alpha_{k}}-\frac{\partial R_{n}(\alpha)}{\partial \alpha_{k}}\right) \\
= & 2 \int_{0}^{\tau}\left\langle\sqrt{n}(\hat{F}(t)-F(t)) u_{n}(\alpha)\right\rangle_{n}\left\langle\frac{\partial u_{n}(\alpha)}{\partial \alpha_{k}} \hat{F}(t)\right\rangle_{n} \lambda(d t) \\
& +2 \int_{0}^{\tau}\left\langle F(t) u_{n}(\alpha)\right\rangle_{n}\left\langle\frac{\partial u_{n}(\alpha)}{\partial \alpha_{k}} \sqrt{n}(\hat{F}(t)-F(t))\right\rangle_{n} \lambda(d t) .
\end{aligned}
$$

The terms on the right-hand side of (10) are considered separately.

Since the Kaplan-Meier estimators are strongly and uniformly consistent, the partial derivatives $\left(\partial / \partial \alpha_{k}\right) u_{j}^{n}(\alpha)$ are bounded, and $n_{i} / n \rightarrow h_{i}>0$ for all $i$, we obtain

$$
\sup _{t \in[0, \tau]}\left|\left\langle\frac{\partial u_{n}(\alpha)}{\partial \alpha_{k}} \hat{F}(t)\right\rangle_{n}-\left\langle\frac{\partial u_{n}(\alpha)}{\partial \alpha_{k}} F(t)\right\rangle_{n}\right|=\sup _{t \in[0, \tau]}\left|\left\langle\frac{\partial u_{n}(\alpha)}{\partial \alpha_{k}}(\hat{F}(t)-F(t))\right\rangle_{n}\right| \rightarrow 0
$$

almost surely as $n \rightarrow \infty$.

On the other hand, one can use (11) and (9) to prove that

$$
\left\langle\left.\frac{\partial u_{n}(\alpha)}{\partial \alpha_{k}}\right|_{\alpha=\theta} F(t)\right\rangle_{n}=0
$$

for all $n \geq 1$. Below we show that this result implies that the first term in (10) tends to zero almost surely.

Consider the second term. Since $\Delta_{n}(\alpha) \rightarrow \alpha_{*}^{T} S \alpha_{*}=\Delta(\alpha)$ as $n \rightarrow \infty$ and $\Delta(\alpha)>0$, there are vectors $\bar{u}(\alpha)$ and $\partial \bar{u}(\alpha) / \partial \alpha_{k}, k=1, \ldots, K$, whose components are

$$
\bar{u}_{j}(\alpha)=\lim _{n \rightarrow \infty} u_{j}^{n}(\alpha)=\frac{1}{\sqrt{\Delta(\alpha)}} \sum_{l=0}^{K} \alpha_{l}\left(\mathbb{I}_{\{l=j\}}-h_{l}\right)
$$

and

$$
\frac{\partial \bar{u}_{j}(\alpha)}{\partial \alpha_{k}}=\lim _{n \rightarrow \infty} \frac{\partial u_{j}^{n}(\alpha)}{\partial \alpha_{k}},
$$

respectively, $j=0, \ldots, K+1$. This implies that

$$
\left\langle F(t) u_{n}(\alpha)\right\rangle_{n}=\sum_{j=0}^{K+1} \frac{n_{j}}{n} F_{j}(t) u_{j}^{n}(\alpha) \rightarrow \sum_{j=0}^{K+1} h_{j} F_{j}(t) \bar{u}_{j}(\alpha), \quad n \rightarrow \infty .
$$

The following result can be used to prove the asymptotic normality of the KaplanMeier estimator for a fixed $j$. Recall that $T_{i j}$ denotes the lifetime for the object $i$ of the sample $j$, while $U_{i j}$ is its (potential) censoring time. 
Theorem 3.1 (4 4, A corollary to Theorem 6.1.3]). Assume that the functions

$$
\pi_{j}(t)=\mathrm{P}\left\{\min \left(T_{i j}, U_{i j}\right) \geq t\right\}
$$

and $F_{j}(t)$ are continuous for $t \geq 0$ and the limits $n_{j} / n \rightarrow h_{j}>0, n \rightarrow \infty$, exist. Let $\Pi_{j}=\left\{t: \pi_{j}(t)>0\right\}$ and let $W$ be the Wiener process. Then

$$
\sqrt{n}\left(\hat{F}_{j}(\cdot)-F_{j}(\cdot)\right) \Longrightarrow\left(1-F_{j}(\cdot)\right) W\left(v_{j}(\cdot)\right), \quad n \rightarrow \infty,
$$

in the Skorokhod space $D[0, t]$ for all $t \in \Pi_{j}$, where

$$
v_{j}(t)=\int_{0}^{t} \frac{d F_{j}(s)}{\pi_{j}(s)\left(1-F_{j}(s)\right)}
$$

and

$$
\operatorname{cov}\left(\left(1-F_{j}(s)\right) W\left(v_{j}(s)\right),\left(1-F_{j}(t)\right) W\left(v_{j}(t)\right)\right)=\left(1-F_{j}(s)\right)\left(1-F_{j}(t)\right) v_{j}(s)
$$

for $0 \leq s<t$.

It is easy to check that the latter theorem holds for all $j$. Therefore

$$
\sum_{j=0}^{K+1} \frac{n_{j}}{n} \sqrt{n}\left(\hat{F}_{j}(t)-F_{j}(t)\right) u_{j}^{n}(\alpha) \Longrightarrow \sum_{j=0}^{K+1} h_{j}\left(1-F_{j}(t)\right) W\left(v_{j}(t)\right) \bar{u}_{j}(\alpha), \quad n \rightarrow \infty .
$$

For an arbitrary vector $a=\left(a_{0}, \ldots, a_{K+1}\right)^{T}$ let

$$
\langle a\rangle=\sum_{j=0}^{K+1} h_{j} a_{j}
$$

Then

and

$$
\left\langle F(t) u_{n}(\alpha)\right\rangle_{n} \rightarrow\langle F(t) \bar{u}(\alpha)\rangle, \quad n \rightarrow \infty
$$

$$
\begin{aligned}
\left\langle\sqrt{n}(\hat{F}(t)-F(t)) u_{n}(\alpha)\right\rangle_{n} & \Longrightarrow\langle(\mathbf{1}-F(t)) \bar{W}(v(t)) \bar{u}(\alpha)\rangle, \\
\left\langle\frac{\partial u_{n}(\alpha)}{\partial \alpha_{k}} \sqrt{n}(\hat{F}(t)-F(t))\right\rangle_{n} & \Longrightarrow\left\langle\frac{\partial \bar{u}(\alpha)}{\partial \alpha_{k}}(\mathbf{1}-F(t)) \bar{W}(v(t))\right\rangle, \\
n & \rightarrow \infty,
\end{aligned}
$$

where $\bar{W}(v(t))=\left(W\left(v_{0}(t)\right), \ldots, W\left(v_{K+1}(t)\right)\right)$ and $\mathbf{1}=(1, \ldots, 1)^{T}$ is the vector constituted of the $(K+2)$ units.

Integration in the space $D[0, \tau]$ is a continuous functional and moreover

$$
\mathrm{P}\left\{\int_{0}^{\tau}\langle(\mathbf{1}-F(t)) \bar{W}(v(t)) \bar{u}(\alpha)\rangle<\infty\right\}=1
$$

and

$$
\mathrm{P}\left\{\int_{0}^{\tau}\left\langle\frac{\partial \bar{u}(\alpha)}{\partial \alpha_{k}}(\mathbf{1}-F(t)) \bar{W}(v(t))\right\rangle<\infty\right\}=1
$$

Thus the first term in (10) weakly converges to

$$
2 \int_{0}^{\tau}\langle(\mathbf{1}-F(t)) \bar{W}(v(t)) \bar{u}(\theta)\rangle\left\langle\left.\frac{\partial \bar{u}(\alpha)}{\partial \alpha_{k}}\right|_{\alpha=\theta} F(t)\right\rangle \lambda(d t)=0
$$

if $\alpha=\theta$, while the second term converges to

$$
2 \int_{0}^{\tau}\langle F(t) \bar{u}(\theta)\rangle\left\langle\frac{\partial \bar{u}(\alpha)}{\partial \alpha_{k}}(\mathbf{1}-F(t)) \bar{W}(v(t))\right\rangle \lambda(d t)
$$


as $n \rightarrow \infty$. Thus

$$
\left.\sqrt{n}\left(\frac{\partial \hat{R}_{n}(\alpha)}{\partial \alpha_{k}}-\frac{\partial R_{n}(\alpha)}{\partial \alpha_{k}}\right)\right|_{\alpha=\theta} \Longrightarrow \zeta_{k}(\theta), \quad n \rightarrow \infty,
$$

where

$$
\zeta_{k}(\theta)=2 \int_{0}^{\tau}\langle F(t) \bar{u}(\theta)\rangle\left\langle\left.\frac{\partial \bar{u}(\alpha)}{\partial \alpha_{k}}\right|_{\alpha=\theta}(\mathbf{1}-F(t)) \bar{W}(v(t))\right\rangle \lambda(d t) .
$$

The random variable $\zeta_{k}(\theta)$ is Gaussian and such that $\mathrm{E} \zeta_{k}(\theta)=0$. Applying the CramérWold device [7] we prove the weak convergence of the vector $\sqrt{n}\left(\hat{R}_{n}^{\prime}(\theta)-R_{n}^{\prime}(\theta)\right)$.

Now we evaluate the covariance matrix of the vector $\zeta(\theta)^{T}=\left(\zeta_{1}(\theta), \ldots, \zeta_{K}(\theta)\right)^{T}$. By definition,

$$
\begin{aligned}
\zeta_{k}(\theta) & =2 \int_{0}^{\tau}\langle F(t) \bar{u}(\theta)\rangle\left\langle\left.\frac{\partial \bar{u}(\alpha)}{\partial \alpha_{k}}\right|_{\alpha=\theta}(\mathbf{1}-F(t)) \bar{W}(v(t))\right\rangle \lambda(d t) \\
& =\left.2 \int_{0}^{\tau}\langle F(t) \bar{u}(\theta)\rangle \sum_{j=0}^{K+1} h_{j} \frac{\partial \bar{u}_{j}(\alpha)}{\partial \alpha_{k}}\right|_{\alpha=\theta}\left(1-F_{j}(t)\right) W\left(v_{j}(t)\right) \lambda(d t) \\
& =\left.2 \sum_{j=0}^{K+1} \frac{\partial \bar{u}_{j}(\alpha)}{\partial \alpha_{k}}\right|_{\alpha=\theta} \int_{0}^{\tau}\langle F(t) \bar{u}(\theta)\rangle h_{j}\left(1-F_{j}(t)\right) W\left(v_{j}(t)\right) \lambda(d t) .
\end{aligned}
$$

Let the components of the vector $\eta(\theta)$ be defined by

$$
\eta_{j}(\theta)=\int_{0}^{\tau}\langle F(t) \bar{u}(\theta)\rangle h_{j}\left(1-F_{j}(t)\right) W\left(v_{j}(t)\right) \lambda(d t), \quad j=0, \ldots, K+1 .
$$

We also put

$$
U(\theta)=2\left(\left.\frac{\partial \bar{u}_{j}(\alpha)}{\partial \alpha_{k}}\right|_{\alpha=\theta}\right)_{k=1, j=0}^{K, K+1}
$$

Then

$$
\zeta(\theta)=U(\theta) \eta(\theta)
$$

and

$$
\operatorname{cov} \zeta(\theta)=U(\theta)(\operatorname{cov} \eta(\theta)) U^{T}(\theta)
$$

This implies that the entry $(i, j)$ of the covariance matrix of the vector $\eta(\theta)$ is

$$
\begin{array}{r}
\int_{0}^{\tau} \int_{0}^{\tau}\langle F(s) \bar{u}(\theta)\rangle\langle F(t) \bar{u}(\theta)\rangle h_{i}\left(1-F_{i}(s)\right) h_{j}\left(1-F_{j}(t)\right) \mathrm{E}\left(W\left(v_{i}(s)\right) W\left(v_{j}(t)\right)\right) \lambda(d s) \lambda(d t) \\
=\mathbb{I}_{\{i=j\}} \int_{0}^{\tau} \int_{0}^{\tau} h_{i}^{2}\langle F(s) \bar{u}(\theta)\rangle\langle F(t) \bar{u}(\theta)\rangle\left(1-F_{i}(s)\right)\left(1-F_{i}(t)\right) \\
\times \min \left(v_{i}(s), v_{i}(t)\right) \lambda(d s) \lambda(d t),
\end{array}
$$

since the Kaplan-Meier estimators constructed from different samples are independent. Hence

$$
\mathrm{E} W\left(v_{i}(t)\right) W\left(v_{j}(s)\right)=0
$$

for $i \neq j$ and all $t$ and $s$. Therefore, the covariance matrix of $\eta(\theta)$ is diagonal. 


\section{BIBLIOGRAPHY}

1. E. L. Kaplan and P. Meier, Nonparametric estimation from incomplete observations, J. Amer. Statist. Assoc. 53 (1958), 457-481. MR0093867 (20:387)

2. D. W. Hosmer and S. Lemeshow, Applied Survival Analysis: Regression Modeling of Time to Event Data, J. Wiley \& Sons, Inc., New York, 1999. MR1674644 (2000c:62172)

3. D. R. Cox and D. Oakes, Analysis of Survival Data, Chapman \& Hall/CRC, London, 1984. MR751780 (86f:62170)

4. D. P. Harrington and T. R. Fleming, Counting Processes and Survival Analysis, J. Wiley \& Sons, Inc., New York, 1991. MR1100924 (92i:60091)

5. R. E. Maŭboroda, Statistical Analysis of Mixtures, Kyiv University, Kyiv, 2003. (Ukrainian)

6. Q. Yu and L. Li, On the strong uniform consistency of the product limit estimator, Sankhya 56 (1994), 416-430.

7. P. Billingsley, Convergence of Probability Measures, John Wiley \& Sons, Inc., New York, 1968. $\operatorname{MR} 0233396(38: 1718)$

Department of General Mathematics, Faculty for Mechanics and Mathematics, National Taras Shevchenko University, Academician Glushkov Avenue 6, Kyiv 03127, Ukraine

E-mail address: ryzhov@univ.kiev.ua

Received 15/FEB/2006

Translated by N. SEMENOV 